\title{
Síndrome en espejo con miocardiopatía no compactada en la madre y el feto. Reporte de caso
}

\section{Minrror syndrome with noncompaction cardionyopathy in the mother and fetus. Case report}

Jesús Arnulfo Velásquez-Penagos, MD, MSc ${ }^{1,2}$; Ana María Flórez-Ríos, MD, MSc ${ }^{1}$; Edison Muñoz-Ortiz, MD, MSc ${ }^{1,2}$; Jairo Alfonso Gándara-Ricardo, MD, MSc ${ }^{1,2}$; Juan Pablo

Flórez-Muñoz, $M D, M S c^{2}$; Erika Holguín-González, $M D, M S c^{2}$

Recibido: 16 de marzo del 2021/Aceptado: 9 de julio del 2021

\section{RESUMEN}

Objetivo: reportar el caso de una gestante con síndrome en espejo asociada a miocardiopatía no compactada (MNC), tanto en la madre como el feto, en los que el tratamiento médico antenatal en la madre llevó a un resultado materno perinatal favorable.

Presentación del caso: se describe el caso de una primigestante de 16 años, con 33 semanas de embarazo, remitida desde una institución de primer nivel de atención a una institución privada de cuarto nivel en la ciudad de Medellín, Colombia, por presentar feto con hidropesía en ultrasonido obstétrico de control. Durante la hospitalización, la paciente presentó signos clínicos y ecocardiográficos de falla cardiaca (disnea, edema e hipoxemia), a la vez que se confirmó el diagnóstico de Hydrops fetalis (síndrome en espejo). Se instauró tratamiento diurético con furosemida en la madre, logrando mejoría del cuadro materno y del edema fetal. En el puerperio mediato hospitalario se confirmaron la presencia de miocardiopatía no compactada en la resonancia magnética nuclear cardiaca, tanto de la madre como del recién nacido. Ambos egresaron

* Correspondencia: Jesús Arnulfo Velásquez-Penagos. jesus.velasquez@udea.edu.co

1. Facultad de Medicina Universidad de Antioquia, Medellín (Colombia)

2. Hospital Universitario San Vicente Fundación, Medellín (Colombia). en adecuadas condiciones y fueron vinculados al programa de seguimiento cardiovascular: falla cardiaca y de cardiopatía congénitas, respectivamente. Conclusión: se presenta un caso de síndrome en espejo asociado a miocardiopatía no compactada materna y fetal. Es limitado el número de reportes de síndrome en espejo por anomalías cardiacas (maternas y fetales) y pobre la descripción de los tratamientos realizados que surgen como temas a investigar. Sería importante considerar el diagnóstico de MNC en fetos con hidropesía no asociados a isoinmunización y con disfunción cardiaca, así como su atención por equipos multidisciplinarios.

Palabras clave: edema fetal, cardiomiopatías, enfermedades placentarias, "no compactación aislada del miocardio ventricular".

\section{ABSTRACT}

Objective: To report the case of a pregnant woman with mirror syndrome associated with noncompaction cardiomyopathy in the mother and the fetus, in which antenatal medical treatment provided to the mother resulted in a favorable perinatal maternal outcome.

Case presentation: A 16-year old primigravida with 33 weeks of gestation referred from a Level I institution to a private Level IV center in Medellín, 
Colombia, because of a finding of fetal hydrops on obstetric ultrasound. During hospitalization, the patient showed clinical and ultrasonographic signs of heart failure (dyspnea, edema and hypoxemia), with the diagnosis of hydrops fetalis (mirror syndrome) also confirmed. Diuretic treatment with furosemide was initiated in the mother, with subsequent improvement of the maternal condition as well as of the fetal edema. During the subacute postpartum period in the hospital, the presence of non-compaction cardiomyopathy was confirmed on cardiac nuclear magnetic resonance imaging in both the mother and the newborn. After discharge in adequated condition, they were included in the cardiovascular follow-up program for heart failure and congenital heart disease, respectively.

Conclusion: A case of mirror syndrome associated with maternal and fetal non-compaction cardiomyopathy is presented. There is a limited number of reports on mirror syndrome due to cardiac anomalies (maternal and fetal), with weak treatment descriptions, pointing to the need for research in this area. It would be important to consider the diagnosis of non-compaction cardiomyopathy in fetuses with hydrops unrelated to isoimmunization or cardiac dysfunction, and approach these cases from a multidisciplinary perspective.

Keywords: Hydrops fetalis, cardiomyopathies, placental diseases, isolated noncompaction of the ventricular myocardium.

\section{INTRODUCCIÓN}

En 1892, John Ballantyne demostró una asociación entre el hidrops fetal, el edema placentario y el edema materno, describiendo el síndrome que lleva su nombre, también conocido como síndrome en espejo (SE) o triple edema (1). Antes de 1970 se creía que el hydrops fetalis era solo consecuencia de isoinmunización Rhesus; sin embargo, en los últimos años se han publicado reportes de caso de este síndrome secundario a gestaciones múltiples, malformaciones fetales, anomalías cardiacas fetales (tales como anomalía de Ebstein y arritmias), tumores fetales y placentarios e infecciones virales (2). El SE es descrito como una condición infrecuente. En un hospital general de referencia en China se informó una incidencia de 0,01\% (3). El SE ha sido informado hasta en el 23\% de las gestaciones donde se evidencia hydrops fetalis (4).

No es clara la fisiopatología del SE, pero se manejan las siguientes teorías.

a. Relación con la preeclampsia. Dado que comparte un cuadro clínico similar a la preeclampsia se considera que mecanismos similares pueden estar involucrados, tales como la disfunción placentaria y el desequilibrio entre factores angiogénicos y antiangiogénicos, mediado por la elevación de "Soluble fms-like tyrosine kinase-1" (sFlt-1)(5,6). El edema y la vellositis placentarias inducirían un estímulo hipóxico que llevaría a mayor liberación de factores antiangiogénicos como el sflt1, del cual se conoce su papel como depresor miocárdico y está relacionado con desenlaces cardiovasculares (7).

b. Elevación de la gonadotrofina coriónica $\mathrm{Hu}-$ mana. Se han descrito niveles excesivamente elevados de B-HCG cuando se comparan con gestaciones normales de similar edad gestacional, relacionándose con el incremento en los edemas y la sobrecarga de volumen $(8,9)$. A diferencia de la preeclampsia, en el SE hay evidencia de hemodilución, no de hemoconcentración $(2,5)$.

c. Teoría del estrés cardiaco fetal. Los péptidos natriuréticos, que incluyen el auricular tipo A (A natruretic Peptide, ANP en inglés), liberado desde las aurículas, el tipo B (B natruretic Peptide, BNP en inglés), liberado desde los ventrículos, y el C-NP (C natruretic Peptide, CNP en inglés), producido en SNC, están involucrados en la regulación del agua corporal, tanto en las gestante como en el feto (10). Son liberados en respuesta a sobrecarga de volumen y de presión miocárdicas. En teoría, la falla cardiaca fetal induce mayor liberación de 
péptidos natriuréticos (BNPy ANP), los cuales pueden tener efecto en la fisiología materna. El BNP fetal cruza la placenta y es resistente a la degradación enzimática placentaria, por tanto, es más estable en plasma (11). Por otro lado, el ANP puede ser producido directamente en la placenta, y está relacionado con los cambios hemodinámicos fetales (11). Cuando se han comparado los niveles de $\mathrm{N}$-terminal proBNP (NT-proBNP), forma precursora e inactiva del BNP en fetos con hydrops no inmune, solo se encuentra elevado de manera significativa en aquellos de origen cardiaco (12). Además, se han encontrado mayores niveles de ANP y BNP en el corazón fetal que en adultos, los cuales pueden tener una mayor funcionalidad ante los estímulos que desencadenan su liberación (13).

El manejo de este síndrome se fundamenta en la identificación de la causa fetal y el tratamiento de la causa del hidrops $(14,15)$. Se han descrito terapias específicas adicionales a la trasfusión intrauterina (en casos de anemia fetal por isoinmunización Rhesus), tales como la reducción y/o ablación con láser de comunicaciones vasculares placentarias en gestaciones gemelares monocoriales con síndrome de transfusión feto fetal (16); tratamiento farmacológico con digoxina para falla cardiaca fetal en anomalía de Ebstein (2), derivaciones peritoneo-amnióticas (casos de masas pélvicas fetales) (17), amniodrenaje $(2,16)$; sin embargo, la tasa de sobrevida fetal en general es baja, tan solo del 32\% (16). Desafortunadamente hasta en el 50\% de los casos no se logra establecer la causa del hidrops, y definitivamente el pronóstico depende de la causa de este (18).

Respecto a la miocardiopatía no compactada (MNC), condición también conocida como ventrículo no compacto (VNC), es una rara cardiomiopatía congénita, descrita inicialmente por Grant en 1926 (19); Towbin la describe como "un trastorno caracterizado por prominentes trabéculas, recesos intratrabeculares y un miocardio ventricular izquierdo con dos capas distintas: compactado y no compactado. Existe continuidad entre la cavidad ventricular izquierda y los recesos intratrabeculares profundos, ambos llenos de sangre, y no se observa evidencia de comunicación con el sistema de la arteria coronaria epicárdica" (20). El VNC afecta principalmente al ventrículo izquierdo, también ocurre no compactación aislada del ventrículo derecho y biventricular (21). La ocurrencia familiar se observa en un 40\% con variabilidad en el genotipo y fenotipo (puede evidenciarse disfunción ventricular sin demostrarse la "no compactación", lo que favorece la explicación dada por algunos autores del espectro clínico amplio de esta condición (22). En cuanto a su frecuencia, puede identificarse en el $0,26 \%$ de los pacientes referidos para ecocardiografía (23). Afecta entre 8 y 12 personas por millón al año, aunque su incidencia puede ser mayor, puesto que los asintomáticos no se diagnostican (22).

Su etiología es desconocida, tradicionalmente se ha asumido una alteración en el proceso de compactación cardiaca en la etapa embrionaria, que ocurre entre la quinta y octava semana; sin embargo, reportes de caso lo controvierten y abren la posibilidad a que también puede ser una condición adquirida en la etapa fetal. Esta controversia se manifiesta en su clasificación, ya que la Asociación Americana del Corazón la clasifica como una miocardiopatía genética (24), mientras que la Sociedad Europea de Cardiología y la Organización Mundial de la Salud la clasifica dentro de la categoría "miocardiopatía familiar/genética no clasificada" $(25,26)$.

Su diagnóstico puede realizarse tanto en la etapa prenatal como en la infancia y la etapa adulta. En la etapa prenatal, Sun y Cols reportan una incidencia de 0,07\% (27). El diagnóstico antenatal es difícil, está basado en la ecocardiografía fetal, Tian L. afirma que "en diagnóstico por ultrasonido cardiaco requiere experticia y estar familiarizado con el diagnóstico de la condición para su reconocimiento" (28). En cuanto al diagnóstico postnatal, en un estudio australiano de cardiomiopatías congénitas en menores de 10 años, la MNC explicó el 9,2\% de los casos (29). En la etapa adulta, la incidencia parece menor; en el registro Europeo de Cardiomiopatías congénitas, el 4,1\% correspondió a casos de MNC (30). No se identificaron datos acerca de su presentación en mujeres gestantes. 
Para aportar información sobre la presencia de esta condición en gestantes presentamos el caso de una gestante en el que identificamos la miocardiopatía no compacta tanto en la madre como en el feto, con un cuadro clínico de falla cardiaca materna y fetal, en el contexto de hidrops fetal y síndrome en espejo, con evolución favorable de la gestante y el recién nacido.

\section{PRESENTACIÓN DEL CASO}

Mujer de 16 años primigestante, con 33 semanas de embarazo, fue remitida en traslado no regulado desde una institución pública de nivel medio de complejidad de atención al Hospital Universitario San Vicente Fundación, centro de referencia de alta complejidad ubicado en Medellín, el cual atiende pacientes de los regímenes contributivo de los trabajadores y subsidiado por el estado en el Sistema de seguridad Social en Colombia, por presentar amenaza de parto pretérmino, y por hallazgos de ecografía gestacional realizada en la institución de segundo nivel, llevada a cabo por un especialista en ginecología y obstetricia, quien reportó "macrosomía fetal, polihidramnios y hallazgos compatibles con ascitis fetal", hemoclasificación $0(+)$. No tenía antecedentes familiares o quirúrgicos de importancia. En el sitio de remisión la paciente recibió esquema de maduración pulmonar completo con Betametasona (12 mg intramusculares cada 24 horas por 2 días, y se le prescribió Nifedipino 30 mg cada 12 horas).

Los signos vitales de la paciente al ingreso a urgencias de nuestra institución fueron: presión arterial (PA): 115/70 mmHg; frecuencia respiratoria (FR): 16 respiraciones por minuto; pulso (P): 96 latidos por minuto; saturación de oxígeno (SatO2) al aire ambiente: 98\%; peso: $72 \mathrm{Kg}$. La auscultación cardiaca inicial fue normal. Altura uterina $35 \mathrm{~cm}$, feto en situación longitudinal, presentación cefálica, fetocardia 125 latidos por minuto. Presentaba 1 contracción en 10 minutos, de mala intensidad y de 25 segundos de duración. Al tacto vaginal: cuello posterior cerrado, longitud: $3 \mathrm{~cm}$, sin salida de líquido por vagina. Feto flotante, pelvis ginecoide. La paciente se hospitalizó para ampliar estudios fetales y maternos, y se continuó medicando con Nifedipino de $30 \mathrm{mg}$ cada 12 horas.

Posteriormente es valorada por los especialistas del programa para atención de gestantes con cardiopatía, grupo multidisciplinario conformado por cardiología, ginecobstetricia, anestesia, enfermería, y neonatología que funciona desde el año 2015. La ecografía gestacional realizada por especialista en medicina materno fetal mostró "peso fetal en percentil 97, placenta anterior derecha, ILA 34,2 (polihidramnios), corazón fetal: válvula tricuspídea displásica, presenta insuficiencia severa con regurgitación hasta de $320 \mathrm{~cm} / \mathrm{s}$, paredes de aurícula derecha hieperecogénicas, derrame pleural derecho, líquido libre en la cavidad abdominal, ductus venoso con aumento de la pulsatilidad en relación con falla cardiaca fetal". Por parte de medicina fetal, se conceptuó mal pronóstico intra y extrauterino. Se descartó isoinmunización Rhesus (se confirmó hemoclasificación O positivo). Las pruebas serológicas para sífilis, toxoplasmosis, citomegalovirus, rubeola, herpes, fueron negativas. Dado el pronóstico fetal se decidió suspender uteroinhibición y permitir progresión de trabajo de parto. La paciente siguió sin contracciones uterinas; sin embargo, a las 36 horas la paciente presentó edemas, disnea (FR: 32), hipoxemia: SatO2: 88\% al aire ambiente y congestión pulmonar (crépitos en ambos campos pulmonares). En ese momento se auscultó soplo cardiaco, holosistólico, III/VI, en focos de la punta, segundo ruido pulmonar reforzado; ante diagnóstico presuntivo de edema pulmonar de etiología aún desconocida, se trasladó a unidad de cuidados especiales maternos y se inició tratamiento con oxígeno suplementario (Venturi al 50\%) y 40 mg intravenosos (IV) de furosemida, presentando mejoría sintomática en la siguiente hora. Se solicitó ecocardiografía transtorácica de manera urgente, la cual fue realizada por cardiólogo, especialista en imágenes cardiacas y con amplia experiencia en gestantes. La ecocardiografía evidenció "hipocinesia global, cavidades izquierdas dilatadas, fracción de expulsión del ventrículo izquierdo disminuida (FEVI 44\%), 
insuficiencia mitral y tricuspídea severas”. Se consideró gestante con falla cardiaca, NYHA IV, estadio $\mathrm{C}$, de etiología no clara, con riesgo cardiovascular y perinatal de presentar complicaciones, utilizando la clasificación OMS modificada (OMSm), categoría IV (alta probabilidad de complicaciones serias, incluida la muerte de un 40-100\%) (31), con indicación de realizar resonancia nuclear magnética (RNM) cardiaca en el postparto. Ante el diagnóstico de hidrops fetal no inmune, de etiología no clara, con hallazgos de cardiopatía fetal no clasificable y de mal pronóstico perinatal, se decidió optimizar y priorizar el manejo de la condición materna sobre la condición fetal e iniciar inducción de parto, dado que la vía del parto no condicionaría el bienestar fetal. Se le explicaron a la paciente las consideraciones realizadas y firmó el consentimiento para suspender el embarazo. El examen vaginal mostró un índice de Bishop de 0, se inició maduración cervical dinoprostona de 10 mg intravaginal. De manera simultánea se optimizó el manejo de la falla cardiaca materna con furosemida 40 mg IV cada 4 horas. Después de 36 horas la paciente presentó mejoría de los edemas y de la disnea, pero sin cambios en el cuello uterino, con el fin de evitar inducción prolongada se decidió realizar cesárea, la cual fue llevada a cabo sin complicaciones. Se obtuvo recién nacida viva, de 2.200 gr de peso, apgar 7-9, sin signos de edemas, se evidencia líquido amniótico superior normal durante el procedimiento. La paciente se trasladó a unidad de cuidados intensivos para continuar vigilancia del puerperio, allí recibió carvedilol, enalapril y tromboprofilaxis con enoxaparina y permaneció 48 horas, evolucionó satisfactoriamente y fue dada de alta 7 días postparto.

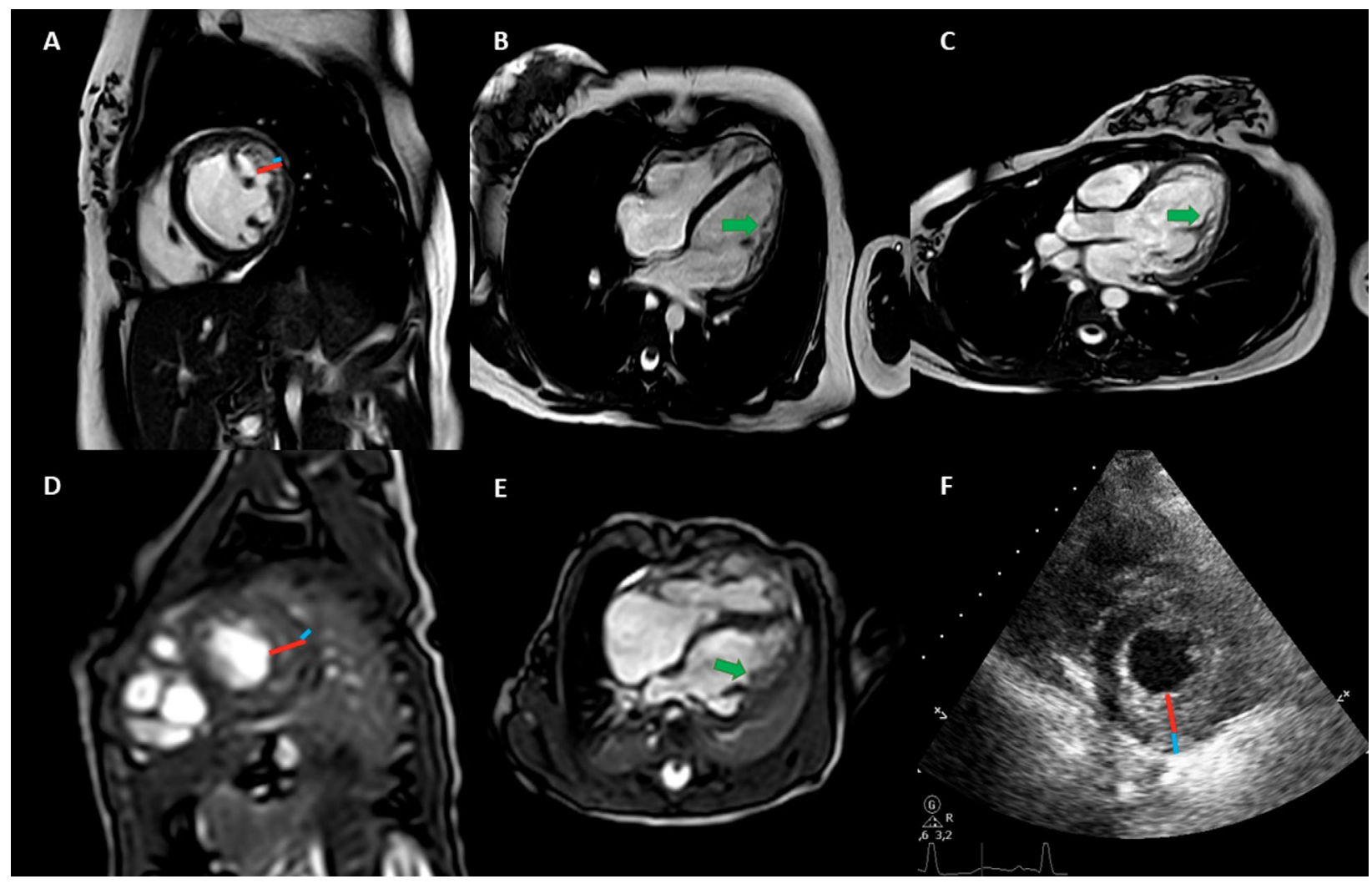

Figura 1. A. Resonancia de la madre: secuencia cine eje corto, línea roja zona no compactada, línea azul miocardio compacto, relación de 2,5. B. Resonancia de la madre: secuencia cine cuatro cámaras, flecha verde indica zona de no compactación en pared anterolateral. C. Resonancia de la madre: secuencia cine tres cámaras, flecha verde indica zona de no compactación en pared inferolateral. D. Resonancia de hijo de la paciente, secuencia cine eje corto, línea roja zona no compactada, línea azul miocardio compacto, relación de 2,7. E. Resonancia de hijo de la paciente, secuencia cine cuatro cámaras, flecha verde indica zona de no compactación en pared anterolateral. F. Ecocardiograma de la madre: eje corto en sístole, línea roja zona no compactada, línea azul miocardio compacto, relación de 2,2. 
La recién nacida se hospitalizó en UCI neonatal, fue evaluada por cardiología pediátrica y genética. La ecocardiografía postnatal inicialmente mostró hipertensión pulmonar (HTP) y disfunción biventricular, por lo que se inició manejo con milrinone. A las 72 horas se repitió ecocardiografía, que mostró menos HTP, pero persistencia de la disfunción biventricular, por lo que se solicitó RNM cardiaca. Toleró desmonte de milrinone y se inició captopril e hidroclorotiazida. La RNM cardiaca materna evidenció criptas endomiocárdicas y signos de no compactación, signos de miocarditis aguda sin fibrosis (ver Figura 1A, 1B, 1C, y 1F). La RNM cardiaca neonatal mostró los mismos hallazgos de ventrículo izquierdo no compacto sin miocarditis (ver Figura 1D y 1E). La recién nacida fue dada de alta en el día 16 postnatal con tratamiento de falla cardiaca iniciado durante la hospitalización. Tanto la madre como la bebé se vincularon a seguimiento por cardiología adultos y pediátricos, respectivamente, en su aseguradora.

Aspectos éticos. Se obtuvo autorización del comité de ética médica institucional. Se garantizó la confidencialidad de la información y la privacidad de la paciente.

\section{DISCUSIÓN}

En este reporte se presenta un caso de síndrome en espejo secundario a una miocardiopatía no compactada (MNC), tanto en la madre como en el feto. El cuadro inició con hydrops fetalis y posterior edema pulmonar materno, con buena evolución materna y perinatal, asociado al tratamiento de la falla cardiaca materna con furosemida. En ambos sujetos el diagnóstico se logró por medio de RM, por cambios estructurales que no fueron visualizados en la ecocardiografía materna fetal o neonatal. Los hallazgos no son consistentes con los descritos por Tian (28), quien considera que el diagnóstico antenatal está basado en la ecocardiografía fetal.

Hasta donde se conoce, este es el primer caso de SE asociado a la MNC presente tanto en la madre como en el feto, y este caso estaría a favor de ser una condición familiar. La percepción de ser el primer caso reportado resulta de la búsqueda de tres de los autores sobre estudios que describieran la asociación del síndrome en espejo por ventrículo no compacto en la madre y el neonato, y que describieran tratamiento y desenlaces perinatales en las bases de datos Medline via PubMed y EMBASE hasta octubre de 2020, con los términos "Left ventricular non compaction", "mirror syndrome”, "pregnancy" y "fetal non compaction", sin restricción de idioma. Se buscaron revisiones sistemáticas de la literatura, series de casos y reportes de caso. Se identificaron 242 títulos, de los cuales se descartaron 235 por no estar relacionados con el objetivo de la revisión, y se incluyeron 7 estudios que describían la presencia de MNC fetal o materna y SE o hydrops fetalis. En tres series de casos, con 183 casos de Síndrome en espejo $(2,4,16)$, se identificaron 4 casos de cardiopatía fetal, pero ninguna de ventrículo no compacto en el feto. Por otra parte, se encontró una de serie de casos en 2015 que identificó 88 fetos con ventrículo no compacto, en los cuales se logró el diagnóstico antenatal en 85, y en 29 (33\%) de estos hubo diagnóstico de hydrops fetalis. Cuatro madres tenían ventrículo no compacto, pero en ninguna se describió síndrome en espejo (32). Posterior a esta publicación se identificaron otros 3 reportes de VNC en gestantes (33-35), sin compromiso fetal por esta condición.

Surge como hipótesis una posible acción sinérgica entre los péptidos natriuréticos maternos y fetales que lleven al SE en la MNC materna y fetal de posible origen familiar. Esto basado en una sumatoria de acciones de los péptidos natriuréticos de origen fetal, placentario y materno, que se ha descrito en los casos de SE de origen cardiaco (tanto anomalías estructurales como las del ritmo cardiaco fetal) (12). Por otra parte, se observa que el buen resultado materno y perinatal posiblemente estuvo asociado al uso de furosemida en la madre, lo que surge como una alternativa terapéutica a evaluar en pacientes con SE (36). 


\section{CONCLUSIÓN}

Este sería el primer caso reportado de síndrome en espejo desencadenado por miocardiopatía no compactada materna y fetal con los términos y en las bases de datos empleados. Es limitado número de reportes de síndrome en espejo por anomalías cardiacas (maternas y fetales) y pobre la descripción de los tratamientos realizados, que surgen como temas a investigar. Es importante considerar el diagnóstico de MNC en fetos con hidrops fetalis no asociados a isoinmunización y con disfunción cardiaca, así como su atención por equipos multidisciplinarios.

\section{REFERENCIAS}

1. Ballantyne JW. The disease and deformities of the fetus. Oliver \& Boyd, editor. Edinburgh; 1892.

2. Braun T, Brauer M, Fuchs I, Czernik C, Dudenhausen JW, Henrich W, et al. Mirror Syndrome: A Systematic Review of Fetal Associated Conditions, Maternal Presentation and Perinatal Outcome. Fetal Diagn Ther. 2010;27(4):191203. https://doi.org/10.1159/000305096

3. Zhao Y, Liu G, Wang J, Yang J, Shen D, Zhang X. Mirror syndrome in a Chinese hospital: diverse causes and maternal fetal features. J Matern Neonatal Med. 2013;26(3):254-8. https://doi.org/10.3109/14767058 .2012 .733765

4. Chen R, Liu M, Yan J, Chen F, Han Q, Zheng L, et al. Clinical characteristics of mirror syndrome: a retrospective study of 16 cases. J Obstet Gynaecol (Lahore). 2020;1-4. https://doi.org/10.1080/01443615.2020.17 18621

5. Hobson SR, Wallace EM, Chan YF, Edwards AG, Teoh MWT, Khaw AP-L. Mirroring preeclampsia: the molecular basis of Ballantyne syndrome. J Matern Neonatal Med. 2020;33(5):768-73. https://doi.org/10.1080/1476705 8.2018 .1500550

6. Goa S, Mimura K, Kakigano A, Tomimatsu T, KinugasaTaniguchi Y, Endo M, et al. Normalisation of Angiogenic Imbalance after Intra-Uterine Transfusion for Mirror Syndrome Caused by Parvovirus B19. Fetal Diagn Ther. 2013;34(3):176-9. https://doi.org/10.1159/000348778

7. Gruson D, Hermans MP, Ferracin B, Ahn SA, Rousseau MF. Sflt-1 in heart failure: relation with disease severity and biomarkers. Scand J Clin Lab Invest.
2016;76(5):411-6. https://doi.org/10.1080/00365513 .2016 .1190863

8. Gherman RB, Incerpi MH, Wing DA, Goodwin TM. Ballantyne syndrome: is placental ischemia the etiology? J Matern Fetal Med. 1998;7(5):227-9. https://doi. org/10.3109/14767059809020448

9. Hirata G, Aoki S, Sakamaki K, Takahashi T, Hirahara F, Ishikawa H. Clinical characteristics of mirror syndrome: a comparison of 10 cases of mirror syndrome with nonmirror syndrome fetal hydrops cases. J Matern Fetal Neonatal Med. 2016;29(16):2630_4. https://doi.org/1 $0.3109 / 14767058.2015 .1095880$

10. Bie P. Natriuretic Peptides and Normal Body Fluid Regulation. In: Comprehensive Physiology. Hoboken, NJ, USA: John Wiley \& Sons, Inc.; 2018. p. 1211-49. https://doi.org/10.1002/cphy.c180002

11. Miyoshi T, Hosoda H, Miyazato M, Kangawa K, Yoshimatsu J, Minamino N. Metabolism of atrial and brain natriuretic peptides in the fetoplacental circulation of fetuses with congenital heart diseases. Placenta. 2019;83:26-32. https://doi.org/10.1016/j. placenta.2019.06.382

12. Lee SM, Jun JK, Kim SA, Kang MJ, Song SH, Lee J, et al. $\mathrm{N}$-terminal pro-B-type natriuretic peptide and cardiac troponin $\mathrm{T}$ in non-immune hydrops. J Obstet Gynaecol Res. 2016 Apr;42(4):380-4. https://doi.org/10.1111/jog.12920

13. Cameron VA, Ellmers LJ. Minireview: Natriuretic Peptides during Development of the Fetal Heart and Circulation. Endocrinology. 2003;144(6):2191-4. https:// doi.org/10.1210/en.2003-0127

14. Chimenea A, García-Díaz L, Calderón AM, Heras MM-D Las, Antiñolo G. Resolution of maternal Mirror syndrome after succesful fetal intrauterine therapy: a case series. BMC Pregnancy Childbirth. 2018;18(1):85. https://doi.org/10.1186/s12884-018-1718-0

15. Nassr AA, Shamshirsaz AA, Belfort MA, Espinoza J. Spontaneous resolution of mirror syndrome following fetal interventions for fetal anemia as a consequence of twin to twin transfusion syndrome. Eur J Obstet Gynecol Reprod Biol. 2017;208:110-1. https://doi.org/10.1016/j. ejogrb.2016.11.023

16. Allarakia S, Khayat HA, Karami MM, Aldakhil AM, Kashi AM, Algain AH, et al. Characteristics and management of mirror syndrome: a systematic review (1956-2016). J Perinat Me. 2017;45(9). https://doi.org/10.1515/jpm2016-0422 
17. Livingston JC, Malik KM, Crombleholme TM, Lim F-Y, Sibai BM. Mirror syndrome: a novel approach to therapy with fetal peritoneal-amniotic shunt. Obstet Gynecol. 2007;110(2 Pt 2):540-3. https://doi.org/10.1097/01. AOG.0000275259.03301.b2

18. Kosinski P, Krajewski P, Wielgos M, Jezela-Stanek A. Nonimmune Hydrops Fetalis_-Prenatal Diagnosis, Genetic Investigation, Outcomes and Literature Review. J Clin Med. 2020;9(6):1789. https://doi.org/10.3390/ jcm9061789

19. Grant R. An unusual anomaly of the coronary vessels in the malformed heart of a child. Heart. 1926;13:273-83.

20. Towbin JA, Lorts A, Jefferies JL. Left ventricular non-compaction cardiomyopathy. Lancet. 2015;386(9995):81325. https://doi.org/10.1016/S0140-6736(14)61282-4

21. Fazio G, Lunetta M, Grassedonio E, Gullotti A, Ferro G, Bacarella D, et al. Noncompaction of the Right Ventricle. Pediatr Cardiol. 2010;31(4):576-8. https://doi. org/10.1007/s00246-010-9652-6

22. Murphy RT, Thaman R, Blanes JG, Ward D, Sevdalis E, Papra E, et al. Natural history and familial characteristics of isolated left ventricular non-compaction. Eur Heart J. 2005;26(2):187-92. https://doi.org/10.1093/eurheartj/ ehi025

23. Sandhu R, Finkelhor RS, Gunawardena DR, Bahler RC. Prevalence and Characteristics of Left Ventricular Noncompaction in a Community Hospital Cohort of Patients with Systolic Dysfunction. Echocardiography. 2007;25(1):8-12 https://doi.org/10.1111/j.15408175.2007.00560.x

24. Maron BJ, Towbin JA, Thiene G, Antzelevitch C, Corrado D, Arnett D, et al. Contemporary definitions and classification of the cardiomyopathies: an American Heart Association Scientific Statement from the Council on Clinical Cardiology, Heart Failure and Transplantation Committee; Quality of Care and Outcomes Research and Functio. Circulation. 2006;113(14):1807-16. https:// doi.org/10.1161/CIRCULATIONAHA.106.174287

25. Elliott P, Andersson B, Arbustini E, Bilinska Z, Cecchi F, Charron P, et al. Classification of the cardiomyopathies: a position statement from the European Society Of Cardiology Working Group on Myocardial and Pericardial Diseases. Eur Heart J. 2008 ;29(2):270-6. https:/doi. org/10.1093/eurheartj/ehm342
26. Richardson P, McKenna W, Bristow M, Maisch B, Mautner B, O'Connell J, et al. Report of the 1995 World Health Organization/International Society and Federation of Cardiology Task Force on the Definition and Classification of cardiomyopathies. Circulation. 1996;93(5):841-2. https://doi.org/10.1161/01. CIR.93.5.841

27. Sun H, Hao X, Wang X, Zhou X, Zhang Y, Liu X, et al. Genetics and Clinical Features of Noncompaction Cardiomyopathy in the Fetal Population. Front Cardiovasc Med. 2020;7:617561. https://doi.org/10.3389/ fcrm.2020.617561

28. Tian L, Zhou Q, Zhou J, Zeng S, Cao D, Zhang M. Ventricular non-compaction cardiomyopathy: prenatal diagnosis and pathology. Prenat Diagn. 2015;35(3):221-7. https://doi.org/10.1002/pd.4523

29. Nugent AW, Daubeney PEF, Chondros P, Carlin JB, Cheung M, Wilkinson LC, et al. The epidemiology of childhood cardiomyopathy in Australia. N Engl J Med. 2003;348(17):1639-46. https://doi.org/10.1056/ NEJMoa02 1737

30. Charron P, Elliott PM, Gimeno JR, Caforio ALP, Kaski JP, Tavazzi L, et al. The Cardiomyopathy Registry of the EURObservational Research Programme of the European Society of Cardiology: baseline data and contemporary management of adult patients with cardiomyopathies. Eur Heart J. 2018;39(20):1784-93. Disponible en: http://www.ncbi.nlm.nih.gov/pubmed/29378019

31. Regitz-Zagrosek V, Roos-Hesselink JW, Bauersachs J, Blomström-Lundqvist C, Cífková R, De Bonis M, et al. 2018 ESC Guidelines for the management of cardiovascular diseases during pregnancy. Eur Heart J. 2018;39(34):3165-241. Disponible en: https://academic.oup.com/eurheartj/article/39/34/3165/5078465

32. Stöllberger C, Wegner C, Finsterer J. Fetal Ventricular Hypertrabeculation/Noncompaction: Clinical Presentation, Genetics, Associated Cardiac and Extracardiac Abnormalities and Outcome. Pediatr Cardiol. 2015;36(7):1319-26. https://doi.org/10.1007/ s00246-015-1200-y

33. Kilic ID, Tanriverdi H, Evrengul H, Uslu S, Sungur MA. Left ventricular non-compaction in pregnancy : case report - online article. Cardiovasc J Afr. 2013;24(3):e1-2. https://doi.org/10.5830/CVJA-2012-075 
34. Kayançiçek H, Alataş Ö, Doğan ÖF. De novo left ventricular non-compaction accompanied by severe mitral valve failure in a young pregnant. Turk gogus kalp damar cerrahisi Derg. 2018;26(2):292-5. https:// doi.org/10.5606/tgkdc.dergisi.2018.15676

35. Gherbesi E, Bonanomi C, Bottari V, Danzi GB. Ventricular tachycardia triggered by pregnancy in left-ventricular non-compaction cardiomyopathy: a controversial indication to automated defibrillator implantation. Monaldi Arch Chest Dis. 2020;90(3). https://doi.org/10.4081/monaldi.2020.1280

36. Beermann B, Groschinsky-Grind M, Fåhraeus L, Lindström B. Placental transfer of furosemide. Clin Pharmacol Ther. 1978 Nov;24(5):560-2. https://doi. org/10.1002/cpt1978245560

\section{FINANCIACIÓN}

Los autores no recibieron ninguna fuente de financiación.

\section{PRESENTACIÓN EN CONGRESOS}

Presentado parcialmente como póster en el XXXI Congreso Nacional de Obstetricia y Ginecología. OPO-30. Cartagena, 2018.

\section{CONTRIBUCIÓN DE LOS AUTORES}

Jesús Arnulfo Velásquez-Penagos: concepción y diseño, adquisición de los datos e información, análisis e interpretación de los datos; planeación del artículo, revisión de contenido intelectual, aprobación final de la versión enviada del artículo.

Ana María Flórez-Ríos: concepción y diseño, adquisición de los datos e información, análisis e interpretación de los datos; planeación del artículo, revisión de contenido intelectual, aprobación final de la versión enviada del artículo.

Edison Muñoz-Ortiz: adquisición de los datos e información, análisis e interpretación de los datos; planeación del artículo, revisión de contenido intelectual, aprobación final de la versión enviada del artículo.

Jairo Alfonso Gándara-Ricardo: adquisición de los datos e información, análisis e interpretación de los datos; planeación del artículo, revisión de contenido intelectual, aprobación final de la versión enviada del artículo.

Juan Pablo Flórez-Muñoz: adquisición de los datos e información, edición de figura, texto explicativo de figura, revisión de contenido intelectual, aprobación final de la versión enviada del artículo.

Erika Holguín-González: análisis e interpretación de los datos; planeación del artículo, revisión de contenido intelectual, aprobación final de la versión enviada del artículo. 